RECAI Revista de Estudios en Contaduría, Administración e Informática

ISSN: 2007-5278

danielarturoc@yahoo.com.mx

Universidad Autónoma del Estado de México

México

\title{
Empresa familiar y la teoría de sistemas luhmanniana
}

Vera Muñoz, Gerardo; Martínez Méndez, Rafaela; Vera Muñoz, María Antonieta Empresa familiar y la teoría de sistemas luhmanniana

RECAI Revista de Estudios en Contaduría, Administración e Informática, vol. 9, núm. 24, 2020

Universidad Autónoma del Estado de México, México

Copyright 2019 Universidad Autónoma del Estado de México

Esta obra está bajo una Licencia Creative Commons Atribución-NoComercial-SinDerivar 4.0 Internacional. 
Artículos

\section{Empresa familiar y la teoría de sistemas luhmanniana}

Family business and the theory of luhmannian systems

Gerardo Vera Muñoz

Benemérita Universidad Autónoma de Puebla, México

Recepción: 02 Septiembre 2019

gerver61@yahoo.com.mx

Rafaela Martínez Méndez

Benemérita Universidad Autónoma de Puebla, México

rafaela72280@hotmail.com

María Antonieta Vera Muñoz

Benemérita Universidad Autónoma de Puebla, México

monseveram@hotmail.com

\section{RESUMEN:}

El objetivo de este trabajo es analizar algunos aspectos de la problemática de la empresa familiar tomando como referencia los conceptos de diferenciación funcional, acoplamiento estructural y complejidad de la teoría de sistemas de Niklas Luhmann. La investigación es eminentemente teórica. Para llevarla a cabo, se revisaron fuentes secundarias como artículos y libros sobre la empresa familiar y la teoría de sistemas luhmanniana. Posteriormente, apoyados en los fundamentos de esta teoría, se formulan una serie de proposiciones que coadyuvan a tener un conocimiento más puntual sobre la problemática de la empresa familiar. Partiendo de la idea de que una organización es un sistema social que se constituye a través de la comunicación de decisiones, se propone que la problemática de la empresa familiar esencialmente tiene sus orígenes en la confrontación cotidiana de códigos de comunicación radicalmente opuestos. Asimismo, se presume que estas unidades empresariales debido a su limitada complejidad: escases de recursos y capacidades; enfrentan graves problemas para adaptarse a su entorno. O, debido a sus propias determinaciones y operaciones internas, no tienen ningún interés en perpetuar tal adaptación.

Palabras ClaVE: empresa familiar, sistemas autopoiéticos.

\section{Abstract:}

The objective of this research work was to analyze some aspects of the problematics of family businesses. This was done by making reference to the concepts of functional differentiation, structural coupling, and complexity of Niklas Luhmann's system theory. The research is eminently theoretical. To carry it out, secondary sources such as articles and books on family business and Luhmannian systems theory were reviewed. Subsequently, supported by the foundations of this theory, a series of proposals were formulated that help to have more precise knowledge about the problematics of the family business. Taking the idea of an organization as a social system constituted through the communication of decisions as a departing point, it is proposed that the problem of the family business essentially has its origins in the daily confrontation of radically opposed communication codes. Likewise, it is presumed that these business units, due to their limited complexity: scarcity of resources and capacities; face serious problems to adapt to their surroundings. Or, down to their determinations and internal operations, they have no interest in perpetuating such adaptation.

KEYWORDS: family business, autopoietic systems.

\section{INTRODUCCIÓN}

Mientras que la empresa grande y, sobre todo, la no familiar ha sido ampliamente estudiada, y se han propuesto herramientas diversas para su gestión, la empresa familiar (en adelante EF) ha sido escasamente analizada, relegada a un segundo plano y catalogada como una organización que se distingue más por sus 
conflictos constantes que por su capacidad para generar y acumular riqueza. Sólo recientemente, al reconocer la importancia que esta unidad de producción tiene en la economía, sobre todo, para la creación de empleo, las investigaciones sobre sus formas de gestión han empezado a proliferar. Por ejemplo, en Francia, el 60.5\% de las empresas son familiares, en Italia el 75\%, en Alemania el 82\%, en el Reino Unido el 76\%, en Estado Unidos el 90\%, en América Latina el 70\%; en el caso específico de México se estima que el 90\% de las empresas excluyendo a la micro empresa, son familiares, y generan el 67\% del empleo (San Martín y Durán, 2017).

A la fecha, la mayoría de las investigaciones llevadas a cabo sobre estas unidades familiares de producción han tomado como referencia los paradigmas que han funcionado para las empresas grandes no familiares. En estas condiciones, las propuestas para apoyar la gestión de la empresa familiar se han reducido a proponer en términos generales y con argumentos insuficientes, la imperante necesidad de seguir el camino de las grandes corporaciones, fundamentado en el orden, la racionalidad y la formalidad.

Un camino que de manera más específica, señala entre otras cosas, apegarse estrictamente a lo que marca el modelo taylorista - fordista, entiéndase, la producción en masa, profesionalizarse, especializarse, reducir costos a cualquier precio, maximizar las ganancias de los propietarios, alcanzar los mayores niveles posibles de eficiencia, certificar sus procesos de producción y, así una lista interminable de sugerencias que, entre las más recientes, podemos mencionar el uso intensivo de las Tic's, en todas las áreas de la empresa; aunque no se explique porqué ni para qué. En síntesis, la mayoría de las propuestas sobre la gestión de la empresa familiar lo que sugieren, como lo señala Pérez (2010), es meterlas al mismo redil de las organizaciones burocráticas, organizadas y administradas en forma profesional ya que se controlan más fácilmente y son consideradas como la punta de lanza de la modernización.

Por lo tanto, el objetivo de este trabajo es intentar explicar algunos aspectos que forman parte de la problemática de la empresa familiar, apoyándonos en los principios básicos de la teoría de sistemas de Niklas Luhmann.

La razón para utilizar la teoría sistemas luhmanniana como referente para el análisis de la empresa familiar, descansa en la manera radicalmente novedosa en que analiza diferentes fenómenos sociales, entre los cuales se encuentran las organizaciones. Específicamente, partiendo de la idea que, las organizaciones al ser sistemas autopoiéticos y operativamente cerrados, responden a las perturbaciones del entorno, de acuerdo a sus propios medios y determinaciones. Con ello, se pretende dejar a un lado las propuestas prescriptivas de las teorías clásicas y neoclásicas de la empresa, derivadas de suponer que era posible organizar todo racionalmente y sobre la base de principios (como se citó en Luhmann, 2010).

El trabajo inicia explicando la estrategia metodológica aplicada, continúa con una revisión teórica de algunas de las aportaciones más significativas sobre la empresa familiar, posteriormente se lleva a cabo una descripción de los principios más relevantes de la teoría de sistemas luhmanniana; con estos referentes pasamos al análisis de los temas principales de la problemática de la empresa familiar, finalizando con las conclusiones.

\section{Metodología}

Uno de los aspectos esenciales en la investigación académica es la propuesta metodológica, en ella se expresa el camino que se seguirá para intentar alcanzar los objetivos propuestos. En este trabajo, la investigación desarrollada es eminentemente teórica. Para llevarla a cabo, se revisaron fuentes secundarias como, artículos y libros, respecto al tema de empresa familiar y sobre la teoría de sistemas luhmanniana. Posteriormente, apoyados en los fundamentos de esta teoría, se formulan una serie de proposiciones que eventualmente coadyuven a tener un conocimiento más refinado sobre la problemática de la empresa familiar. Por lo tanto, se puede decir, que la estrategia metodológica es básicamente cualitativa, donde el investigador articulando conceptos, teorías y técnicas de investigación, asume la tarea de darle significación y sentido a la información recolectada (Davila, 1999). 


\section{REvisión DE LA LITERATURA}

\subsection{La empresa familiar}

Una de las investigaciones más amplia y citada sobre empresa familiar es la de Sharma, Chrisman, y Chua (1997). Sin embargo, al tomar como referente el proceso de planeación estratégica cometen un grave error, emplean un concepto que surge en el seno de las grandes corporaciones con condiciones y circunstancias distintas a las empresas familiares, la mayoría de tamaño micro y pequeño y donde quien las administra es también el propietario. Hay que tener presente que el concepto de planeación estratégica surge precisamente, cuando la empresa tiene la necesidad de articular sus objetivos de forma sistemática para su público externo, lo que no ocurría cuando la compañía era propiedad del mismo pequeño grupo de personas que también la administraban (Knights y Morgan, 1991). Algunos de los temas que se discuten sobre la empresa familiar es la relación entre las metas familiares y las metas de la empresa, y por qué se seleccionan determinados objetivos; se aborda el tema de la sucesión, un tema recurrente en cualquier investigación sobre la empresa familiar y, otros aspectos como el gobierno corporativo. Sin embargo, tal vez, lo más significativo de la investigación de Sharma, Chrisman y Chua (1997), es señalar que, en una empresa familiar, la familia y el negocio están tan enredados que las emociones son inevitables. Y que el negocio familiar está menos diferenciado horizontalmente y depende más de los controles informales que las empresas no familiares y, en consecuencia, la empresa familiar puede tener más éxito en escenarios que requieren una estructura más flexible.

Por otra parte, Hollander y Elman (1988), identifican tres enfoques diferentes adoptados por los investigadores para formular modelos evolutivos sobre la empresa familiar. El primer enfoque relaciona las etapas de desarrollo de la empresa con la progresión generacional de la familia (Barnes y Hershon, 1989). El segundo se centra en la interacción entre las necesidades de la empresa y las etapas de vida de las personas cruciales para la empresa (Danco, 1975; Davis y Tagiuri, 1989; McGivern, 1989). El tercero considera la interacción de tres conjuntos de ciclos de vida: la empresa, la familia y los individuos clave (Ward, 1987). El tema subyacente en todos estos modelos es que la delegación de responsabilidades y poder a los miembros no familiares varía significativamente en las diferentes etapas.

Otras investigaciones sobre la empresa familiar, destacan que, no obstante que la gestión del crecimiento es crucial para asegurar el desarrollo sostenido de la empresa, esto no parece ser uno de los objetivos más valorados en el contexto de la empresa familiar (Claver, Rienda, y Quer, 2006). En este mismo orden de ideas, Davis y Stern (1988), señalan que muchas familias que controlan negocios familiares encuentran difícil adaptarse a las demandas de cambio tecnológico y del entorno. Algunas no son capaces de enfrentarse a las adaptaciones organizativas y de la familia requeridas y detienen el proceso evolutivo, a menudo con consecuencias desastrosas.

En una investigación sobre la empresa familiar ucraniana, Brice y Jones (2008) encontraron que la ventaja competitiva de las empresas familiares se basa en la cultura familiar inimitable de su organización. Esta cultura conduce a un ambiente familiar, a una mayor lealtad de los empleados, a una mayor reputación de integridad y comportamiento ético, a una alineación más estrecha entre la cultura y la estrategia de la organización, a una respuesta más rápida a los cambios ambientales y a un punto de vista a largo plazo menos reactivo a los ciclos económicos.

Por otro lado, Astrachan y Kolenko (1994), destacan que un factor descuidado que no ha sido investigado a fondo, que explica el éxito del negocio familiar son las prácticas de recursos humanos. De acuerdo a estos autores la empresa familiar puede construir ventajas competitivas de diversas maneras, pero la esencial es desarrollando las capacidades organizacionales de sus empleados.

Por su parte, Leach (2010) a partir de un estudio realizado en más de 8000 firmas familiares del Reino Unido, subraya que la empresa familiar es la única entidad que puede verdaderamente hacer proyectos a largo plazo, sin embargo, fallan al momento de formalizar sus planes, para describirlos, analizar las conjeturas y 
comparar los resultados con las previsiones. Agrega, que la estabilidad en una empresa familiar es uno de sus originales y más valiosos activos; pero, al mismo tiempo, es necesario pensar si una cultura empresarial estable puede llegar a ser un obstáculo para el cambio y la adaptabilidad. Otro aspecto que destaca, es que familia y empresa son dos campos de acción bastante diferentes. En el ámbito familiar, que se fundamenta en aspectos emocionales, prevalece la protección y la lealtad, mientras que, en la esfera empresarial, sustentada en la tarea, rigen el rendimiento y los resultados.

Dodero (2012), en una investigación llevada a cabo en empresas familiares latinoamericanas, destaca como factores que explican el éxito de estas firmas, la visión estratégica y la dedicación; esto, como resultado de la pasión y el compromiso que ponen los familiares para sacar la empresa adelante, lo que habitualmente sucede cuando quien está al frente es un gran emprendedor con visión de negocio. Por otra parte, encontró que entre los errores más graves que se cometen en las empresas familiares, están problemas organizativos, conflictos por fallas en la comunicación y una administración deficiente.

Una investigación sobre la EF que aporta ideas interesantes es la de Claver, Rienda y Pertusa (2004), enfatiza que cuando una familia funda una empresa generalmente se cometen dos errores. En primer lugar, estos familiares creen que el afecto mutuo basta para afrontar los posibles problemas empresariales que puedan surgir. Y, en segundo lugar, piensan que una empresa familiar se puede equiparar a cualquier otra empresa sin recibir ningún trato especial.

En México, aparte de Soto (2013), que realizó una revisión del estado del arte sobre la empresa familiar, recopilando algunas de las aportaciones más significativas sobre: el fundador y los empleados no familiares, la sucesión, prácticas gerenciales y administración de recursos; otros académicos e investigadores que han abordado la problemática de la empresa familiar son Belausteguigoitia (2003); lo significativo de su obra es señalar la necesidad de entender que empresa y familia son dos sistemas diferentes que ejercen entre si una influencia mutua.

Otra investigación sugestiva sobre la EF en México, es la de López, Contreras, y Molina (2013), destaca el intento de tomar a la EF como objeto de estudio; esta idea se sostiene particularmente en la introducción de la obra, donde se cuestiona la universalidad que se le quiere asignar al concepto de empresa familiar, y se formulan un conjunto de preguntas sobre su problemática.

En este contexto de la empresa familiar en México, Pérez (2010) y su equipo de trabajo, apoyados en una perspectiva antropológica, lograron identificar algunas de las micro relaciones entre familia y empresa. Específicamente, se explica la importancia de las redes sociales para el acceso a recursos y su relación con el capital social. Otro aspecto relevante, de la investigación de Pérez (2010), es haber encontrado que la motivación original de crear las unidades de producción fue el bienestar familiar, no la acumulación de capital.

Apoyándonos en los párrafos anteriores, se puede decir que, por un lado, se observa una clara tendencia a someter el análisis de la problemática de la empresa familiar a la óptica de lo que ha funcionado para las empresas grandes. Como ejemplo, están las investigaciones de Sharma, Chrisman, y Chua (1997), y Dodero (2012), quienes utilizaron la planeación estratégica como marco de referencia; o las investigaciones de Davis y Tagiuri (1989), que se apoyan en la teoría clásica de sistemas y en el enfoque evolutivo. Estas investigaciones enfatizan la importancia de la estrategia, de la racionalidad, de la gestión del crecimiento y la incapacidad de las empresas familiares para apoyar su proceso evolutivo. Por otra parte, están aquellas investigaciones que han intentado estudiar a la empresa familiar como un ente con características propias, pero sin poder deslindarse del todo, de los conceptos sobre el management surgidos de las grandes corporaciones, como ejemplo se puede mencionar a Leach (2010), Claver, Rienda y Pertusa (2004), Belausteguigoitia (2003) y Pérez (2010). Estas investigaciones destacan la importancia de entender que familia y empresa son dos sistemas diferentes, el primero sustentado en aspectos emocionales, que busca el bienestar y la estabilidad familiar; el segundo sustentado en la tarea que persigue el crecimiento, el cambio y la innovación.

\subsection{La Teoría de Sistemas de Niklas Luhmann}


Las aportaciones de Niklas Luhmann se ubican principalmente en el ámbito de la Sociología y de la Comunicación, sin embargo, su obra también abarcó el análisis de las organizaciones tomando como referencia la teoría sociopoiética.

En términos muy básicos, la teoría sociopoiética es una propuesta de Niklas Luhmann que surge de un proyecto que pretende una refundación y reformulación de la teoría sociológica, una comprensión sociológica de la sociedad, y de la modernidad, a partir del andamiaje de la teoría de sistemas y posteriormente de los conceptos de autopoiésis, provenientes de la biología de la cognición (Luhmann, 2007).

Es importante puntualizar que, no obstante, que el mismo Luhmann desarrolló una teoría sociopoiética de la organización, en este trabajo, los referentes que se aplicarán para el análisis de la empresa familiar serán aquellos provenientes de la teoría de sistemas luhmanniana.

La propuesta teórica de luhmann se construye esencialmente a partir de los conceptos de sistema, autopoiésis, clausura operativa y auto/heterorreferencia. Así como de la diferenciación social, el acoplamiento estructural y la complejidad (representaciones). Apoyándonos en Luhmann (2012), empezaremos diciendo, que lo que un sistema es se define por su función. Existen múltiples tipos de sistemas, pero todos ellos tienen una única y misma función; que consiste en reducir complejidad. Dicho en otros términos, en resolver problemas del universo. Ampliando esta idea, se tiene, que en el universo operan ciertos procesos o mecanismos o fuerzas u operaciones que reducen complejidad, esto es, aminoran el desorden, reducen localmente el carácter contingente, indeterminado y problemático del universo. A estos procesos reductores de complejidad se les denomina sistemas.

Con estas ideas, Luhmann (2012) llega a la definición de sistema. Un sistema es una diferencia entre sistema y entorno. Todo sistema posee un entorno, no hay sistema sin entorno, y el entorno es siempre (infinitamente) más complejo que el sistema.

Tomando como referencia la figura 1, se puede observar que hay sistemas de muchos tipos: físicos, químicos, biológicos; que buscan reducir complejidad. Ahora bien, de acuerdo a Luhmann (2012), el hombre no es un sistema y, por lo tanto, para enfrentar diversos problemas de complejidad que le depara la cotidianidad recurre a un recurso que le es único, que lo distingue del resto de los animales. Este recurso es el sentido. El hombre se sirve de él para mediar su relación con el mundo y reducir complejidad; esto significa que gracias al hombre y su recurso el sentido, existen, además de los sistemas físicos, químicos y biológicos, sistemas significativos que operan en base al sentido. Un aspecto que es importante subrayar, es que lo que distingue a los sistemas no es su medio o su recurso, sino la operación que en base al mismo realicen. En el caso de los hombres, se valen del sentido para realizar dos tipos de operaciones significativas: la conciencia, y la comunicación.

Siguiendo con Luhmann (2012) y con la figura 1, es en base a la operación denominada conciencia que surgen lo que el denomina sistemas psíquicos, equivalentes a los sujetos o individuos de la lengua cotidiana. $\mathrm{Y}$ es en base a la comunicación que surgen los sistemas sociales. Propone una tipología de sistemas sociales de acuerdo a distintos tipos de comunicaciones, ordenadas en base a distintos criterios de distinción entre el adentro y el afuera de la comunicación, esto es, entre sistema y entorno comunicativo.

Estos distintos criterios de distinción son: la presencia, la pertenencia y la participación. Y a partir de cada uno de estos criterios surgen tres tipos de sistemas sociales (ver figura 1): las interacciones, las organizaciones y las sociedades. Respecto a las organizaciones, Luhmann coincide con Mumby y Clair (2000), pues para ellos, una organizacio\#n es una colectividad social, que se produce, se reproduce y se transforma por medio de las pra\#cticas de comunicacio\#n habituales, interdependientes y deliberadas de sus miembros. Agregan que las organizaciones sólo existen en la medida en que sus miembros las crean por medio del discurso (para Luhmann es la comunicación de decisiones). Puntualizan que no significa que las organizaciones sean "nada ma\#s" que discurso, sino que este es el principal medio por el cual los miembros de una organizacio\#n crean una realidad social coherente que encuadra la percepcio\#n que tienen de su propia identidad.

\subsubsection{La diferenciación social}


A los distintos tipos de sistemas sociales, hay que agregar los distintos tipos de estructuras sociales, lo que da lugar a la teoría de la diferenciación social, esto es, de los subsistemas sociales. De acuerdo a Luhmann (2012), existen tres tipos básicos de mecanismos de diferenciación social: la segmentación, la estratificación y la diferenciación funcional.

En términos muy simplificados en el caso de la segmentación, los sistemas sociales se diferencian internamente en sub-sistemas que preservan la semejanza con los sub-sistemas del entorno; los sub-sistemas son semejantes entre sí, tanto en estructura interna como en jerarquía.

En lo que respecta a la estratificación, Luhmann (2012), señala que es la diferenciación de un sistema social en sub-sistemas comunicativos que preservan su semejanza estructural interna al precio de una diferencia jerárquica entre sub-sistemas situados unos en el entorno de los otros. Se trata por ello, de un modo de diferenciación que combina la semejanza (intra-sistémica) con la diferencia (inter-sistémica) entre los diversos subsistemas.

Por último, y lo más importante para el análisis que se realizará posteriormente, es lo que respecta a la diferenciación funcional; en este tipo de organización sub-sistémica cada sistema se ordena en base a sus propios criterios diferenciales, pero como todas las funciones diferenciadas sub-sistémicamente son igualmente necesarias para el conjunto de la sociedad, en sus entornos se encuentran frente a subsistemas no jerarquizados o igualitarios. Se trata de un principio de diferenciación que combina diferencias al interior de cada sistema, con semejanzas entre los mismos. La diferenciación funcional es el principio de organización de las sociedades modernas, por lo que, organiza sus principales estructuras comunicativas en términos funcionales (Luhmann, 2012).

Lo que es relevante de esta diferenciación funcional es que cada sub sistema funcional se organiza y autorregula de acuerdo a su propio lenguaje sistémico, o medio de comunicación simbólicamente generalizado, que especifica y selecciona un tipo especial de comunicación. Para la política se trata del medio del poder, para la economía del dinero, para las familias y parejas (sistemas afectivos) del amor, para la ciencia el de la verdad, etcétera. Estos medios de comunicación, a su vez, se basan en un código binario específico (o criterio especificador y selector/ordenador de las respectivas especificaciones): para la ciencia, el código de binarios se basa en la oposición entre verdad y falsedad; para el derecho, el código binario se basa en la oposición entre legal e ilegal; para la religión, en la oposición entre inmanencia y trascendencia; para el arte entre belleza y fealdad, o entre originalidad y mediocridad, etcétera. Esto código puede aplicarse a todo tipo de problemas, ya sea del propio sistema, ya sean del entorno. Así, para la economía monetarizada moderna todo lo que ocurre dentro o fuera de ella sólo puede ser juzgado en términos de rentable/no rentable (o caro/ barato; comprable/no comprable, etcétera).

\subsubsection{El acoplamiento estructural}

Una idea esencial en la teoría de sistemas luhmaniana, es la de sistema auto/hetororreferente, es decir, un sistema que se observa a si mismo y observa cuanto encuentra en su entorno, pudiendo mediante esta operación establecer determinados procedimientos de selección y reducir la complejidad del entorno que le rodea (Luhmann, 1990); se trata de una idea que supone grandes diferencias con respecto al concepto clásico de sistema.

El concepto clásico de sistema precisa que un sistema es un conjunto de elementos que mantienen determinadas relaciones entre sí y que se encuentran separados de un entorno determinado; el sistema se define siempre respecto a un determinado entorno. En cambio, en la teoría de los sistemas auto/ heterorreferentes, el sistema se define, precisamente por su diferencia respecto a su entorno; una diferencia que se incluye en el mismo concepto de sistema. Así, un sistema que contiene en si mismo la diferencia con su entorno, es un sistema auto/heterorreferente y autopoiético.

De acuerdo a la teoría de autopoiésis, elaborada por los biólogos chilenos H. Maturana y D. Varela, un sistema es autopiético en tanto es un sistema que puede crear su propia estructura y los elementos de que se compone (como se citó en Luhmann, 1990). Lo anterior conduce al concepto de clausura operativa, lo 
que significa que los sistemas autopiéticos son sistemas operativamente cerrados, es decir, no toman nada de su entorno, sino que producen y reproducen por si mismos los elementos y estructuras de su consistencia sistémica (Luhmann, 2007).

Al tratarse de sistemas autopoiéticos y operativamente cerrados, es necesario recurrir al concepto de acoplamiento estructural, que responde a la pregunta: ¿cómo es que el sistema entra en relación con el entorno? La respuesta señala que el acoplamiento estructural excluye el que datos existentes en el entorno puedan especificar, conforme a las estructuras, lo que sucede en el sistema. No determina lo que sucede en el sistema, pero debe estar presupuesto, ya que de otra manera la autopiésis se detendría y el sistema dejaría de existir. En este sentido, todos los sistemas están adaptados a su entorno (o no existirían), pero hacia el interior del radio de acción que así se les confiere, tienen todas las posibilidades de comportarse de un modo no adaptado (Luhmann, 2007).

Asimismo, el acoplamiento estructural entre dos sistemas operativamente cerrados opera detonando respuestas, reacciones internas al sistema, irritaciones dentro de un sistema originadas en estímulos extraños provenientes del entorno, que impiden al sistema seguir operando normalmente, por lo que lo obligan a acomodar sus operaciones y estructuras a las perturbaciones del entorno (Luhmann, 2012). Esto es: cada sistema responde en sus propios términos, y según sus propias operaciones, a irritaciones y estímulos ciegos provenientes del entorno. De este modo se ajustan mutuamente los diversos sistemas.

En el caso de la conciencia y la comunicación pueden acoplarse estructuralmente por medio del lenguaje natural, que irrita simultaneamente a la conciencia y a la comunicación, al participar información por medio del sentido. A nivel del sistema social, los diversos sub sistemas funcionalmente diferenciados no pueden comunicarse entre sí para coordinar sus operaciones. Solo pueden acoplar sus estructuras, irritarse mutuamente, provocarse disfunciones recíprocas que los obligan a resolver problemas de funcionamiento interno derivados de obstáculos externos; en el caso de las organizaciones, son el único subsistema capaz de comunicarse con su entorno (Luhmann, 2012, 2010).

\subsubsection{Las representaciones}

Finalmente, sobre la teoría de sistemas luhmanniana mencionaremos el tema de las representaciones. Iniciaremos con el tema de la complejidad. En base a él se puede decir, que el mundo de un sistema significativo (el horizonte de experiencias y acontecimientos abarcable por un sistema que procesa sentido) posee una complejidad paralela a la del propio sistema. Así sistemas sociales poco complejos pueden reducir y procesar escasa complejidad, resolver pocos problemas, lo cual significa que su capacidad de procesar complejidad por medio del sentido es limitada. Como consecuencia, esos sistemas poseen mundos significativos muy poco complejos, su capacidad de resolver problemas es muy limitada, y su imagen del mundo también lo es (se puede decir: un mundo muy poco complejo, y un ultramundo que no se conoce ni se comprende, muy complejo). A medida que incrementa la capacidad de resolver problemas de ese sistema, a medida que se complejiza el sistema, para hacer frente a la complejidad del entorno, su mundo significativo se complejiza más (Luhmann, 2012).

Sin embargo, que el mundo de una sociedad segmentaria o tribal sea un mundo simple, no significa que no sea capaz de percibir la complejidad del universo, de la naturaleza, o de los asuntos humanos. Ese tipo de sociedades perciben la complejidad del mundo, pero no pueden reducirla significativamente. Así, el mundo se les presenta como un caudal inagotable de problemas no resueltos; $y$, en el fondo, como un profundo misterio. A menor capacidad de reducir complejidad por parte de los sistemas significativos (psiquícos y sociales), mayor misterio por parte del mundo, mayor incertidumbre cognitiva, mayor irracionalidad de la acción. De ahí que, que como hay enormes áreas de la realidad incomprensibles e inmanejables, dicha complejidad no reducida del mundo asuma la forma de lo sagrado (Luhmann, 2012).

Para llevar a cabo el análisis de la EF tomando como referente la teoría de sistemas luhmanniana, es necesario especificar, desde ya, apoyándonos en la figura 1, que cualquier organización, sin importar su tamaño, el sector al que pertenezca o la actividad que realicen, es un sistema social basado en la comunicación; 
esto es así, debido a que la teoría luhmanniana introduce en el pensamiento sociológico un cambio fundamental: el concepto de acción es sustituido por el concepto de comunicación como constitución de los hechos sociales. Y esto ha sido posible, porque de acuerdo a su definición solamente la comunicación es siempre un fenómeno social, a diferencia de la acción que es genéricamente individual y requiere ser calificada de social. En el caso específico de las empresas son sistemas sociales basados en la comunicación de decisiones, a través de las cuales se producen, se auto reproducen y se transforman, y que se caracterizan por poner condiciones que deben ser aceptadas por quienes desean pertenecer a ellas (Luhmann, 2010).

Asimismo, otra razón, para abordar la problemática de la EF a partir de la teoría de sistemas luhmanniana, radica, en que es la única que tiene el andamiaje teórico suficiente y necesario para entender y analizar una organización única en su tipo, constituida por dos sistemas radicalmente distintos: el sistema familia y el sistema empresa. A la fecha, como se ha mencionado, las investigaciones sobre la EF han sido realizadas a partir de los postulados de las teorías clásicas y neoclásicas de la empresa, que surgen de concebir a la firma como un sistema único, cuyas decisiones se orientan por la búsqueda del beneficio máximo, de la racionalidad y del orden, que por supuesto, son ideas significativas, pero no suficientes para entender la dinámica de una empresa como la familiar donde tan importante es la lógica económica como la lógica emocional. A esto, hay que agregar, la pérdida de confianza en la posibilidad de organizar racionalmente (en el sentido del mejor modo posible) sobre la base de principios, y esta confianza ha desaparecido tanto en las teorías empresariales de la industria como en la investigación sociológica de la organización. Esto último, resultado de la especialización creciente de la sociología, que condujo a los sociólogos organizacionales a dedicar sus esfuerzos a comprender los sistemas organizacionales (la empresa entre otros) como si estuvieran asilados, esto es, prescindiendo de la sociedad que hacia posible que surgieran, que los rodeaba como entorno, de la cual estaban hechos como sistemas de comunicaciones y a cuya existencia contribuían con sus comunicaciones.

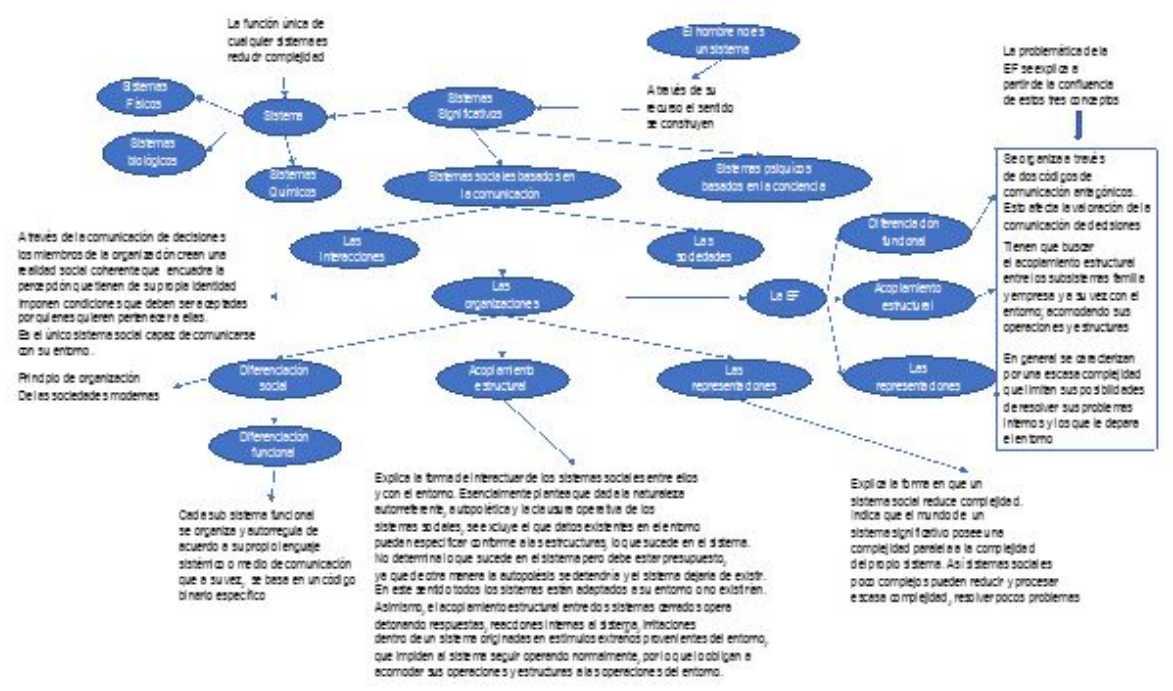

Figura 1. Teoría de sistemas luhmanniana y EF

Fuente: elaboración propia. En esta figura se establecen las relaciones entre los conceptos esenciales de la teoría de sistemas de Niklas Luhmann y, como a partir de algunos de estos conceptos es posible explicar la problemática de la EF.

\section{Algunos aspectos de la PRoblemática de LA EMPRESA FAMILIAR ANALIZAdos DESDE LA PERSPECTIVA DE LA TEORÍA DE SISTEMAS LUHMANNIANA.}

Apoyándonos en la figura 1 iniciaremos por señalar que de acuerdo a la teoría de sistemas luhmanniana, cualquier organización, por ejemplo, una empresa, sin importar su tamaño y si es o no es de tipo familiar, es 
un sistema social basado en el criterio de pertenencia o membresía, que busca reducir complejidad y, que se produce, se reproduce y se transforma a partir de la comunicación de decisiones (Luhmann, 2012).

\subsection{Análisis de la diferenciación funcional}

Atendiendo a la diferenciación funcional, en la figura 1 se observa que cada sub sistema funcional se organiza y autorregula de acuerdo a su propio lenguaje sistémico, o medio de comunicación simbólicamente generalizado, que especifica y selecciona un tipo especial de comunicación. Así se tiene que en el subsistema economía, el medio de comunicación simbólicamente generalizado es el dinero, mientras que para el subsistema familia es el amor. En estas condiciones, en la empresa familiar se confrontan medios de comunicación opuestos: dinero versus amor; una lógica racional versus una lógica emocional. Estas ideas explican los hallazgos de Leach (2010); quien menciona que familia y empresa son dos campos de acción bastante diferentes. Mientras que el ámbito familiar se fundamenta en aspectos emocionales, prevaleciendo la protección y la lealtad; en la esfera empresarial, sustentada en la tarea, rigen el rendimiento y los resultados.

Además, hay que tener presente, retomando a Luhman (2012), que estos medios de comunicación, a su vez, se basan en un código binario específico, que para el caso de la empresa el código binario se cimienta en la oposición entre ganancias y pérdidas, entre éxito y fracaso o entre productividad e ineficiencia. Mientras que para la familia su código binario se fundamenta en la oposición entre protección y desamparo, entre afecto e indiferencia. Esto da por resultado que las comunicaciones de decisiones, que son los componentes esenciales de las organizaciones, estén sujetas a juicios con criterios radicalmente distintos; dando por resultado como lo señala Dodero (2012), fallas en la comunicación, problemas organizativos y una administración deficiente. Así como el escaso interés por la gestión del crecimiento y la acumulación del capital, mencionados por Claver, Rienda, y Quer, (2006) y Pérez (2010), respectivamente.

\subsection{Análisis del acoplamiento estructural}

Retomando las ideas de Luhmann $(2007,2012)$, sobre el acoplamiento estructural, apoyándonos en la figura 1, hay que recordar que designa relaciones entre sistema y entorno, que aunque no intervienen determinando estructuralmente en el sistema y, por lo tanto, son compatibles con la autopoiésis, a largo plazo influyen en las estructuras autoproducidas en el sistema mismo y, en este sentido, desencadenan una deriva estructural. Es a través de este acoplamiento estructural que la empresa familiar responde a las perturbaciones que se generan entre el sistema familia, el sistema empresa y con el entorno. Así por ejemplo, es el caso de aquellas empresas familiares en donde modificando sus operaciones y estructuras, conciliando medios y códigos de comunicación radicalmente opuestos, logran construir reglas o protocolos que especifican el perfil de los familiares que en un momento dado pueden ser incorporados a la empresa, o sobre las formas en que se integrará el gobierno de la firma (el caso del gobierno corporativo). En cuanto a las perturbaciones generadas por el entorno, un ejemplo de acoplamiento estructural es cuando aquellas empresas familiares que operaban en la economía informal, modifican sus operaciones internas y deciden integrarse a la economía formal vía un regímen de contribución especial.

Hay que tener presente, que las formas o caminos en que cada subsistema acomoda sus estructuras y operaciones es única, de acuerdo a sus propios recursos y determinaciones. Por ello, estas formas y caminos pueden ser múltiples e incluso novedosos e inesperados.

\subsection{Análisis de las representaciones}

Recurriendo una vez más a la figura 1, y lo mencionado en párrafos anteriores, las representaciones establecen la relación entre el nivel de compeljidad del sistema y su capacidad para reducir y procesar complejidad. En las organizaciones, ya sea al momento de construirlas o reconstruirlas, esto es una de las decisiones fundamentales, expresada en el tamaño del sistema, es decir: al número de puestos de trabajo por eregir. De esto depende la complejidad, en el sentido de la heterogeneidad de puestos que puede alcanzar la organización y de ésta, a su vez, qué problemas de reducción de complejidad deben ser resueltos, vinculando dichos puestos entre si Luhmann (2012). 
Así, en muchas de las empresas familiares, mayormente de tamaño micro y pequeño, con un número máximo de trabajadores, lo que limita la heterogeniedad de puestos que puede alcanzar la organización, con estructuras horizontales escasamente diferenciadas y con un elevado nivel de informalidad, su grado de complejidad es mínimo y, por ello, sólo pueden reducir y procesar escasa complejidad, lo que limita su capacidad para afrontar problemas de su entorno. Perciben las oportunidades y amenazas que les rodean, pero no tienen la capacidad suficiente o el interés para aprovechar las primeras y enfrentar las segundas.

Prefieren renunciar a las oportunidades de mercado que se les presentan, que optimizar sus procesos de producción, mejorar y desarrollar nuevos productos. Prefieren mantener pequeño y constante el tamaño de la empresa y operar únicamente con trabajadores pertenecientes a la familia, que enfrentar un posible incremento de costos de sueldos, problemas de derecho laboral y fiscales.

A lo anterior, se puede agregar que el tema de la complejidad explica lo señalado por Leach (2010), respecto a la dificultad para formalizar planes, para analizar conjeturas y comparar resultados de previsiones. Así como, a la dificultad para adaptarse a las necesidades del cambio tecnológico y del entorno (Davis y Stern, 1988).

\section{CONCLUSiOnes}

Recurriendo a la categoría diferenciación funcional, se puede presumir que los temas principales que constituyen la problemática de la empresa familiar, tienen que ver con aspectos donde se enfrentan dos sistemas que operan con medios y códigos de comunicación radicalmente opuestos. Por el lado de la familia, un código sustentado en aspectos emocionales; por el lado de la empresa, un código sustentado en la tarea. Se trata de unidades de producción únicas, en donde conviven dos sistemas antagónicos.

Por otra parte, se puede suponer que las empresas familiares que logran mantenerse en el mercado, ha sido por su capacidad para alcanzar un acoplamiento estructural entre el sistema familia y el sistema empresa y con el entorno; acoplando sus operaciones y estructuras de cada sistema, generando respuestas a las irritaciones que cada sistema produce en el otro. Sin embargo, es necesario agregar que debido a que cada sistema responde en sus propios términos, y según sus propias operaciones, las respuestas son muy diversas e impredecibles, y no responden a ninguna mecánica instructiva. Así, podemos encontrar empresas familiares que guiadas por la busqueda del bienestar familiar, una vez alcanzado este objetivo, no les interese gestionar un mayor crecimiento y mejorar su posición en el mercado. Otras prefieran mantenerse en la economía informal, algunas más incorporar trabajadores ajenos a la familia e iniciar un proceso de profesionalización, etcétera.

Recurriendo a la categoría denominada representaciones, se logró explicar el por qué las empresas familiares, particularmente las micro y pequeñas, que se pueden catalogar como sistemas poco complejos, debido entre otras cosas, a su tamaño, número mínimo de puestos de trabajo y la heterogeniedad en los puestos de trabajo que puede alcanzar la organización, enfrentan tantas dificultades para procesar y reducir la compeljidad del entorno. Sin embargo, esto no significa que estas empresas no perciban la complejidad del escenario empresarial, la perciben, pero no pueden reducirla significativamente y, a muchas de ellas, no les interesa enfrentar esta complejidad.

Es pertinente insistir, en la necesidad de entender que cada empresa familiar, sin importar su tamaño es única, y que sus posibilidades de mejora dependen exclusivamente de sus procesos internos. De aquí se desprende la banalidad que ha caracterizado a muchas de las propuestas de tipo prescriptivo derivadas de las teorías clásicas de la firma.

Finalmente, se puede presumir, apoyándonos en las referencias anteriores que la empresa familiar desde su génesis surge con una clara desventaja ante las empresas no familiares, al tener que acoplar dos sistemas que, debido a su diferenciación funcional, se basan en medios y códigos de comunicación radicalmente distintos y, a su vez, acoplarse con el entorno. Un entorno de enorme complejidad que, dada la naturaleza de la mayoría de las empresas familiares, sistemas sociales poco complejos, sus posibilidades de alcanzar tal acoplamiento son limitadas. 


\section{ReFERENCIAS}

Astrachan, J. y Kolenko, T. (1994). A neglected factor explaining family business success: Human resource practices, Familiy Business Review, 3(7), 251-262.

Barnes, B. y Hershon, A. (1989). Transferring power in the family business, Family Business Review, 2(2), 187-202.

Belausteguigoitia, I. (2003). Empresas Familiares su Dinámica, Equilibrio y Consolidación. México: Mc Graw Hill.

Brice, W. y Jones, W. (2008). The cultural foundations of family business, Eurasian Journal of Business and Economics, $1(1), 3-23$.

Claver, E., Rienda, L. y Pertusa, E. (2004). Un marco teórico para la continuidad de la empresa familiar desde un punto de vista estratégico, ESIC MARKET, 118, 229-257.

Claver, E., Rienda, L. y Quer, D. (2006). El comportamiento de las empresas familiares y no familiares en los mercados extranjeros: un estudio comparativo, Cuadernos de Gestión, 6(2), 11-25.

Danco, L. (1975). Beyond Survival: A Business Owner's Guide for Success. Cleveland, OH: University Press.

Dávila, A. (1999). Las perspectivas metodológicas cualitativa y cuantitativa en las ciencias sociales: Debate teórico e implicaciones praxeológicas, en Delgado, J. y Gutiérrez, J. (Coords.), Métodos y Técnicas Cualitativas de Investigación en Ciencias Sociales, 69-83. Madrid, España: Síntesis.

Davis, P. y Stern, D. (1988), Adaptation, survival, and growth of the family business: An integrated systems perspective, Family Business Review, 1(1), 69-84.

Davis, J. y Tagiuri, R. (1989). The influence of life-stage on father-son work relationships in family companies, Family Business Review, 2(1), 47-74.

Dodero, S. (2012). El Secreto de las empresas familiares exitosas. Buenos Aires, Argentina: Editorial Ateneo.

Hollander, S. y Elman, S. (1988). Family-owned businesses: An emerging field of inquiry. Family Business Review, 1(2), $145-164$.

Knights, D. y Glenn M. (1991). Corporate strategy, organizations, and subjectivity: A critique, Organization Studies, 12(2), 251-273.

Leach, P. (2010). La empresa Familiar. Buenos Aires, Argentina: Granica Editores

López, A., Contreras, R. y Molina, R. (2013). La Empresa Familiar como Objeto de Estudio. Ciudad de México, México: Pearson

Luhmann, N. (1990). Sociedad y Sistema: La Ambición de una Teoria. Barcelona, España: Paidós Ibérica S. A.

Luhmann, N. (2007). Introducción a la Teoría de Sistemas. Ciudad de México, México: Universidad Iberoamericana.

Luhmann, N. (2010). Organización y Decisión. Ciudad de México, México: Universidad Iberoamericana.

Luhmann, N. (2012). El Amor. Buenos Aires, Argentina: Prometeo libros.

McGivern, C. (1989). The dynamics of management succession: A model of chief executive succession in the small family firm, Family Business Review, 2(4), 401-411.

Mumby, D. y Claire, R. (2000). El discurso en las organizaciones. En T. Van Dijk (Comp.), El Discurso Como Interacción Social, 263-298. Barcelona, España: Gedisa.

Pérez, M. (Comp.). (2010).Empresa y Familia en México Una Visión desde la Antropología. Ciudad de México, México: Universidad Iberoamericana.

San Martín, J. y Durán, J. (2017). Radiografía de la Empresa Familiar en México. Puebla: UDLAP

Sharma, P., Chrisman, J. y Chua, J. (1997). Strategic management of the family business: past research and future challenges, Family Business Review, Spring, 10(1), 1-36.

Soto, A. (2013). La empresa familiar en México situación actual de investigación, Contaduría y Administración, 58(2), $135-171$.

Ward, J. (1987). Keeping the Family Business Healthy: How to Plan for Continuing Growth, Profitability, and Family Leadership, San Francisco, CA: Jossey-Bass 
Gerardo Vera Muñoz, et al. Empresa familiar y la teoría de Sistemas luhmanNiana

Copyright 2019 Universidad Autónoma del Estado de México

CC BY-NC-ND 